\title{
Clinical Distinct Features of Noncardiac Chest Pain in Young Patients
}

\author{
Tae Ho Seo, MD ${ }^{1}$, Jeong Hwan Kim, MD ${ }^{1,2 *}$, Jung Hyeon Lee, MD', Soon Young Ko, MD ${ }^{1}$, Sung Noh Hong, MD ${ }^{1,2}$, In-Kyung \\ Sung, $M D^{1}$, Hyung Seok Park, $M D^{1}$, and Chan Sub Shim, $M D^{1}$ \\ ${ }^{1}$ Department of Internal Medicine, Digestive Disease Center, ${ }^{2}$ Medical Immunology Center, Konkuk University School of Medicine, Konkuk \\ University Medical Center, Seoul, Korea
}

\section{Background/Aims}

Noncardiac chest pain (NCCP) is a very common disorder world-wide and gastroesophageal reflux disease (GERD) is known to be the most common cause. The prevalence of NCCP may tend to decrease with increasing age. However, there is little report about young aged NCCP. The aim of this study was to examine the prevalence of GERD and to evaluate the efficacy of proton pump inhibitor (PPI) test in the young NCCP patients.

\section{Methods}

Thirty patients with at least weekly NCCP less than 40 years were enrolled. The baseline symptoms were assessed using a daily symptom diary for 14 days. Esophago-gastro-duodenoscopy (EGD) and $24 \mathrm{hr}$ esophageal $\mathrm{pH}$ monitoring were performed for the diagnosis of GERD and esophageal manometry was done. Then, patients were tried with lansoprazole $30 \mathrm{mg}$ twice daily for 14 days, considering positive if a symptom score improved $\geq 50 \%$ compared to the baseline.

\section{Results}

Nine (30\%) of the patients were diagnosed with GERD at EGD and/or $24 \mathrm{hr}$ esophageal $\mathrm{pH}$ monitoring, also, $3(10 \%)$ were diagnosed with GERD-associated esophageal motility disorder and $3(10 \%)$ were non GERD-associated. Concerning PPI test, GERD-related NCCP had a higher positive PPI test $(n=8,89 \%)$ than non GERD-related NCCP $(n=5,24 \%)(p=0.002)$.

\section{Conclusions}

In young patients with NCCP, a prevalence of GERD diagnosed using EGD and/or $24 \mathrm{hr}$ esophageal pH monitoring was 30\%. PPI test was very predictable on diagnosis of GERD-related NCCP, thus, PPI test in young NCCP patients may assist to the physician's clinical judgment of NCCP.

(J Neurogastroenterol Motil 2010;16:166-171)

\section{Key Words}

Gastroesophageal reflux disease, Noncardiac chest pain, Proton pump inhibitor, Young adult

Received: March 11th, 2010 Accepted: April 1st, 2010

(c) This is an Open Access article distributed under the terms of the Creative Commons Attribution Non-Commercial License (http://creativecommons. org/licenses/by-nc/3.0) which permits unrestricted non-commercial use, distribution, and reproduction in any medium, provided the original work is properly cited.

*Correspondence: Jeong Hwan Kim, MD

Department of Internal Medicine, Digestive Disease Center, Medical Immunology Center, Konkuk University School of Medicine, Konkuk University Medical Center, 4-12 Hwayang-dong, Gwangjin-gu, Seoul 143-729, Korea

Tel: +82-2-2030-5010, Fax: +82-2-2030-5029, E-mail: sefamily@kuh.ac.kr

Financial support: This study was supported by a grant from The Korean Society of Neurogastroenterlogy and Motility, SK Chemicals. Conflicts of interest: None. 


\section{Introduction}

Noncardiac chest pain (NCCP) is defined as recurring angina-like or substernal chest pain believed to be unrelated to the heart after a reasonable cardiac evaluation, ${ }^{1-3}$ and affects approximately one-third of the population during their lifetime. ${ }^{3,4}$ It is a benign condition with an estimated 10 -year mortality of less than $1 \% .^{5}$ However, the associated morbidity, as a result of the inability to work and the related health care utilization, are enormous. ${ }^{6}$

Gastroesophageal reflux disease (GERD) is known to be the most common cause of $\mathrm{NCCP},{ }^{7-9}$ being in up to $60 \%$ of patients with NCCP. ${ }^{10}$ The available diagnostic tests including esophago-gastro-duodenoscopy (EGD), esophageal manometry, $24 \mathrm{hr}$ esophageal $\mathrm{pH}$ monitoring, esophageal $\mathrm{pH}$-impedance monitoring, and an empirical trial with a high-dose proton pump inhibitor (PPI) during a short course. ${ }^{11-14}$

Young age may be one of the potential risk factors for NCCP. ${ }^{15}$ In clinical setting, we have experienced a number of young NCCP cases with the different clinical features. However, the majority of studies on NCCP have not focused on a specific age group. In fact, the importance of NCCP in young patients deserves careful attention considering its influence on their active social life and their low likelihood of cardiac abnormality. ${ }^{16}$ Therefore, there could be a renewal of interest in the individualised diagnosis and management for young adults. However, there has been little report of this aged NCCP.

The aim of this study was to examine the prevalence of GERD in the young patients with NCCP, and to evaluate the clinical characteristics and the usefulness of the empirical trial with PPI.

\section{Materials and Methods}

\section{Patients}

We performed a study for patients with NCCP less than 40 years at the Konkuk University Medical Center, Seoul, Korea, from April 2007 to December 2008. The patients were visited to the coronary unit for at least one episode of unexplained chest pain per week, for a minimum 3 months. After visit to the coronary unit, patients had a normal admission electrocardiogram, no abnormalities of cardiac enzymes, negative treadmill exercise testing, and/or normal or insignificant findings on coronary angiograms. And then, the eligible patients were referred to gastroenterology. Exclusion criteria included severe liver, lung, renal, or hematological disorders, a history of peptic ulcer disease or gastrointestinal surgery, a history of a connective tissue disorder, and chest pain originating from a musculoskeletal disorder. Patients were also excluded if they were already using antireflux medications such as PPI or $\mathrm{H} 2$ receptor blocker and pain modulators such as benzodiazepine, tricyclic antidepressant or selective serotonin reuptake inhibitor, or if they were unwilling or unable to provide informed consent, or if they could not complete all phases of the study. Informed written consent was obtained from all participating patients. This study was approved by the Institutional Review Board of Konkuk University Medical Center.

\section{Study protocol}

For the baseline assessment, the clinical and symptomatic characteristics were investigated by using a structured questionnaire and the upper gastrointestinal tests including EGD, esophageal manometry and ambulatory $24 \mathrm{hr}$ esophageal $\mathrm{pH}$ monitoring were performed. All patients were classified as GERD-related NCCP or non GERD-related NCCP based on the results of EGD and ambulatory $24 \mathrm{hr}$ esophageal $\mathrm{pH}$ monitoring. Documentation of reflux esophagitis on EGD and/or pathologic acid exposure on ambulatory $24 \mathrm{hr}$ esophageal $\mathrm{pH}$ monitoring was used to determine the presence of GERD. Also, the baseline symptom assessment was accomplished using a daily symptom diary for 14 days. Following the baseline assessments, the patients were entered into the therapeutic trial of lansoprazole $30 \mathrm{mg}$ am before breakfast and $30 \mathrm{mg}$ pm before dinner for 14 days. During this period of the lansoprazole trial, the patients continued to keep the same daily symptom diary.

\section{Upper gastrointestinal tests}

\section{1) Esophago-gastro-duodenoscopy}

EGD was conducted by an experienced endoscopist with standard endoscopes (XQ-260, Olympus Optical Co. Ltd., Tokyo, Japan) after an overnight fast. The distal portion of the esophagus was carefully examined to determine the presence of mucosal injury. The extent of esophageal mucosal damage was assessed according to the Los Angeles classification. ${ }^{17}$

\section{2) Esophageal manometry}

Esophageal manometry was undergone before $\mathrm{pH}$-monitoring studies according to current standard guidelines. Esophageal manometry was performed using a standard $4.5 \mathrm{~mm}$ diameter, water-perfused, 6-channel Esophageal manometry catheter 
(Dentsleeve Oesophageal LES sleeve catheter, Medtronics, Minneapolis, MN, USA). The catheter was passed transnasally and positioned with the most distal channel in the stomach, the next most proximal (Dentsleeve, Medtronics, Minneapolis, MN, USA) in the lower esophageal sphincter (LES), and the remaining channels in the esophagus at 5,10,15 and $20 \mathrm{~cm}$ above LES. With the patient supine, five 'wet' swallows, each consisting of $5 \mathrm{~mL}$ of water, were given at intervals of 20 seconds. Peristaltic activity and LES function were observed and if any abnormalities were detected a further five $5 \mathrm{~mL}$ 'wet' swallows were given ('full' manometric study). On completion of the swallows, the catheter was slowly withdrawn, the upper esophageal sphincter position and function observed, and the catheter removed, and a series of 15 wet swallows was performed. The diagnosis of each esophageal motility abnormality was verified, according to the accepted published criteria. ${ }^{18}$

\section{3) Ambulatory $24 \mathrm{hr}$ esophageal $\mathrm{pH}$ monitoring}

Ambulatory $24 \mathrm{hr}$ esophageal $\mathrm{pH}$ monitoring was conducted with a $2.1 \mathrm{~mm}$ diameter monocrystalline $\mathrm{pH}$ catheter equipped with 2 antimony electrodes (Synectics, Irving, TX, USA) separated $15 \mathrm{~cm}$ apart off PPI for 7 days. The catheters were introduced transnasally, in order to position the sensors $5 \mathrm{~cm}$ above the upper edge of the manometrically determined LES. The esophageal acid exposure values (percentage of the time the $\mathrm{pH}$ was $<4.0$ ) were calculated using a commercial software program (EsoPHogram, version 5.70C2, Gastrosoft Inc., Irving, TX, USA). Pathologic acid exposure was defined as an intraesophageal $\mathrm{pH}$ of less than 4 , for more than $4.0 \%$ of the recording time. ${ }^{19}$

\section{PPI test}

For 14 days, patients recorded the frequency and severity of chest pain daily using a daily symptom diary. The severity was expressed in the following 4 grades: mild ( 1 , the symptom was easily tolerated and did not last long); moderate (2, the symptom caused some discomfort, but did not interfere with usual activities); severe (3, the symptom caused a great deal of discomfort, and interfered with usual activities); and disabling (4, the symptom was unbearable and interfered considerably with usual activities). ${ }^{7}$ A symptom intensity score was calculated by adding the reported daily severity multiplied by the reported daily frequency values obtained for 14 days at baseline, then for the same days of the lansoprazole trial. This test was considered positive if the symptom intensity score improved by more than $50 \%$ from the baseline. ${ }^{11}$

\section{Statistical methods}

The Mann-Whitney U test was utilized to examine the difference in the baseline symptom scores between GERD-related $\mathrm{NCCP}$ and non GERD-related NCCP groups. A comparison of the PPI test results (positive or negative) between GERD-related NCCP and non GERD-related NCCP groups was accomplished utilizing the Fisher's Exact Test. A p $<0.05$ was considered significant.

\section{Results}

Forty consecutive patients with NCCP less than 40 years were screened. Among them, 2 had a history of peptic ulcer disease, 2 had a history of a connective tissue disorder, and 6 declined ambulatory $24 \mathrm{hr}$ esophageal $\mathrm{pH}$ monitoring or had technically unsuccessful monitoring. As result, 30 patients were enrolled in this study (17 men and 13 women with a mean age of $32.2 \pm 5.8$ years $)$.

The patients were classified into 2 groups, GERD-related $\mathrm{NCCP}$ and non GERD-related NCCP according to the results

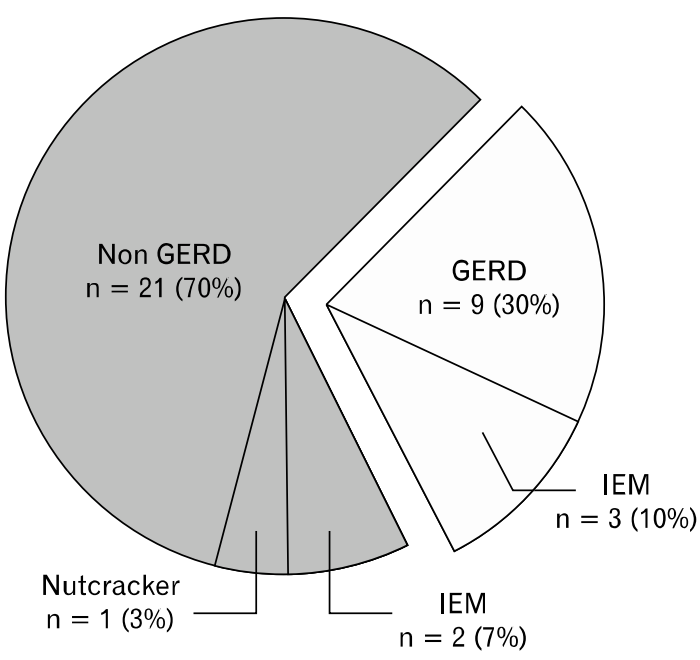

Figure 1. Upper gastrointestinal evaluation. Gastroesophageal reflux disease (GERD)-related noncardiac chest pain (NCCP) was found in nine (30\%) of 30 patients on EGD and/or ambulatory $24 \mathrm{hr}$ esophageal $\mathrm{pH}$ monitoring and esophageal motility disorder was found in 6 patients (20\%) including 5 with ineffective esophageal motility and 1 with nutcracker esophagus. Also, GERD-associated esophageal motility disorder was found in $3(10 \%)$ and non GERD-associated esophageal motility disorder in only 3 patients (10\%). Non GERD, non GERD-related NCCP; Nutcracker, nutcracker esophagus; IEM, ineffective esophageal motility. 
Table 1. Patients' Characteristics

\begin{tabular}{|c|c|c|c|c|}
\hline & & $\begin{array}{l}\text { GERD-related } \\
\operatorname{NCCP}(\mathrm{n}=9)\end{array}$ & $\begin{array}{l}\text { Non GERD-related } \\
\operatorname{NCCP}(\mathrm{n}=21)\end{array}$ & p-value \\
\hline \multicolumn{2}{|l|}{ Age $[$ mean yr $\pm \mathrm{SD}]$} & $33.5 \pm 6.5$ & $31.5 \pm 5.2$ & 0.57 \\
\hline \multicolumn{2}{|l|}{$\operatorname{Sex}[\mathrm{M} / \mathrm{F}]$} & $5 / 4$ & $12 / 9$ & 0.35 \\
\hline \multicolumn{2}{|l|}{$\mathrm{BMI}\left[\mathrm{kg} / \mathrm{m}^{2}\right.$, mean $\left.\pm \mathrm{SD}\right]$} & $22.8 \pm 1.1$ & $23.8 \pm 2.1$ & 0.46 \\
\hline \multicolumn{2}{|l|}{ Smoking $[\mathrm{n}(\%)]$} & $5(42 \%)$ & $7(33 \%)$ & 0.45 \\
\hline \multicolumn{2}{|l|}{ Alcohol $[\mathrm{n}(\%)]$} & $5(42 \%)$ & $8(38 \%)$ & 0.65 \\
\hline \multicolumn{2}{|c|}{ Daily symptom intensity $[$ mean $\pm \mathrm{SD}]$} & $4.61 \pm 4.10$ & $4.54 \pm 3.75$ & 0.91 \\
\hline \multirow[t]{3}{*}{ Symptom frequency $[\mathrm{n}(\%)]$} & $\geq 1 /$ day & $3(33 \%)$ & $7(33 \%)$ & 0.56 \\
\hline & $3 /$ wk $\leq<1 /$ day & $3(33 \%)$ & $9(43 \%)$ & \\
\hline & $1 / \mathrm{wk} \leq<3 / \mathrm{wk}$ & $3(33 \%)$ & $5(24 \%)$ & \\
\hline \multirow[t]{4}{*}{ Symptom duration $[\mathrm{n}(\%)]$} & $3 \mathrm{mo}-6 \mathrm{mo}$ & $2(12 \%)$ & $3(14 \%)$ & 0.32 \\
\hline & $6 \mathrm{mo}-1 \mathrm{yr}$ & $1(11 \%)$ & $3(14 \%)$ & \\
\hline & $1 \mathrm{yr}-5 \mathrm{yr}$ & $4(45 \%)$ & $13(62 \%)$ & \\
\hline & $>5 \mathrm{yr}$ & $2(12 \%)$ & $2(10 \%)$ & \\
\hline \multirow[t]{5}{*}{ Reflux esophagitis [n (\%)] } & & $8(89 \%)$ & $0(0 \%)$ & \\
\hline & LA grade $\mathrm{A}$ & $6(67 \%)$ & & \\
\hline & LA grade B & $1(11 \%)$ & & \\
\hline & LA grade $\mathrm{C}$ & $1(11 \%)$ & & \\
\hline & LA grade D & $0(0 \%)$ & & \\
\hline \multicolumn{2}{|c|}{ Pathologic acid exposure $[\mathrm{n}(\%)]^{\mathrm{a}}$} & $6(67 \%)$ & $0(0 \%)$ & \\
\hline \multicolumn{5}{|c|}{ Esophageal $\mathrm{pH}[$ mean percentage $\pm \mathrm{SD}]$} \\
\hline & $\%$ total time $\mathrm{pH}<4$ & $10.23 \pm 11.05$ & $1.55 \pm 1.23$ & 0.02 \\
\hline & $\%$ upright time $\mathrm{pH}<4$ & $14.53 \pm 13.25$ & $2.35 \pm 2.28$ & 0.01 \\
\hline & $\%$ supine time $\mathrm{pH}<4$ & $2.58 \pm 9.22$ & $0.34 \pm 0.19$ & 0.04 \\
\hline
\end{tabular}

${ }^{\mathrm{a}}$ Pathologic acid exposure, percentage of the time for intraesophageal $\mathrm{pH}<4$ more than $4.0 \%$.

GERD, gastroesophageal reflux disease; NCCP, noncardiac chest pain; BMI, body mass index; LA, Los Angeles classification; NS, not significant. Bold style indicates statistical significance.

of EGD and/or ambulatory $24 \mathrm{hr}$ esophageal $\mathrm{pH}$ monitoring. GERD-related NCCP group included nine patients (30\%). Reflux esophagitis was found in seven patients on EGD and pathologic acid exposure was identified in 6 patients on ambulatory $24 \mathrm{hr}$ esophageal $\mathrm{pH}$ monitoring. Four patients had both reflux esophagitis and pathologic acid exposure. Non GERD-related NCCP group, with 21 patients (70\%), had both normal $\mathrm{EGD}$ and $\mathrm{pH}$ test results. On esophageal manometric examination, esophageal motility disorder was found in 6 patients (20\%). Five of 6 patients had ineffective esophageal motility (IEM) and 1 had nutcracker esophagus. Three of the 5 IEM patients also had GERD (GERD-associated esophageal motility disorder) and the other 3 patients did not have GERD (non GERD-associated esophageal motility disorder) (Fig. 1).

GERD-related NCCP and non GERD-related NCCP groups did not differ significantly in age, gender, mean body mass index (BMI, $\mathrm{kg} / \mathrm{m}^{2}$ ), smoking history, or history of chronic alcoholism. The baseline symptom intensity score for chest pain showed no significant difference between GERD-related NCCP

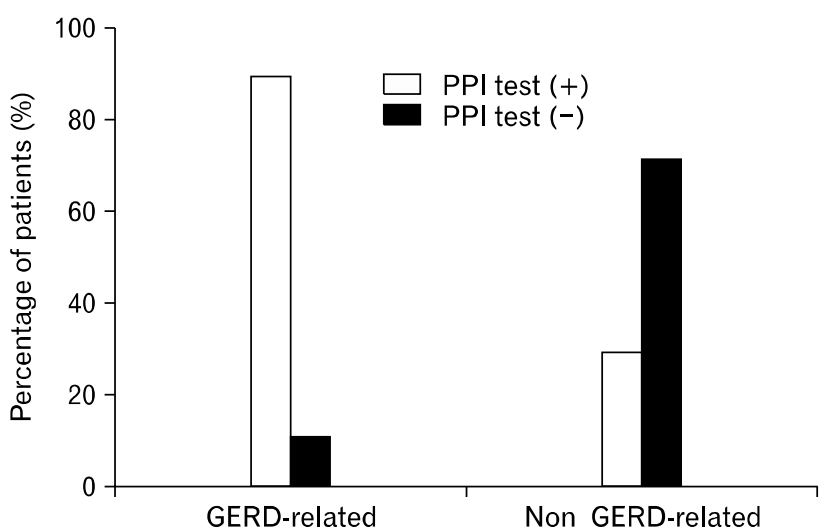

Figure 2. A comparison of proton pump inhibitor (PPI) testing between gastroesophageal reflux disease (GERD)-related noncardiac chest pain (NCCP) and non GERD-related NCCP groups. During the 14 days of the PPI trial, the percent of positive PPI tests was significantly higher in GERD-related NCCP group (89\%) compared to non GERD-related NCCP group (29\%). $\mathrm{p}=$ 0.002 . 
and non GERD-related NCCP groups. The 2 groups also did not differ significantly in the duration or frequency of symptoms (Table 1).

During the 14 days of the PPI trial, 8 patients (89\%) had a positive PPI test ( $>50 \%$ symptom improvement) in GERD-related NCCP group and 6 patients (29\%) had a positive PPI test in non GERD-related NCCP group. This difference was statistically significant $(\mathrm{p}=0.002)$ (Fig. 2).

\section{Discussion}

In this present study, we examined the prevalence of GERD in the patients with NCCP less than 40 years, and to evaluate the usefulness of the empirical trial with PPI. In this uncontrolled trial, $9(30 \%)$ of the patients with $\mathrm{NCCP}$ were diagnosed with GERD at EGD and/or $24 \mathrm{hr}$ esophageal pH monitoring. Also, we found that the 2 weeks PPI trial resulted in symptom improvement in $89 \%$ of GERD-related NCCP patients and this result was statistically significant compared with non GERD-related NCCP patients.

$\mathrm{NCCP}$ is a very common disorder world-wide, presenting in approximately $23-33 \%$ of the population. ${ }^{20,21}$ Several pathophysiological mechanisms have been suggested including GERD, esophageal motility disorders, visceral hyperalgesia, psychiatric disturbances, abnormal cerebral processing of visceral stimulation, and disrupted autonomic activity. ${ }^{22,23}$ The diagnosis and management of patients with NCCP is a frequent and perplexing problem facing clinicians. Most patients continue to present a diagnostic and therapeutic challenge to their primary care physicians after a reasonable cardiac evaluation. These patients are frequently highly debilitated and tend to use a disproportionate level of health care resources, including recurrent doctor and emergency room visits, hospitalizations, and prescription medications, leading them to indicate poor satisfaction with their medical care. $^{24}$

Concerning age factors, the recent report by Eslick et al. ${ }^{3}$ showed that the prevalence of NCCP tended to decrease with increasing age in the population-based study. Moreover, young age may be one of the potential risk factors for NCCP. ${ }^{15}$ In clinical setting, we have experienced a number of young NCCP cases with some different characteristics comparing with average aged NCCP. However, there has been little report about the pathophysiological mechanisms, diagnosis, and management of young aged NCCP. In this present study, we focused on the prevalence of GERD and the diagnostic value of PPI test of the young aged
NCCP patients, exclusively. In NCCP patients less than 40 years, the proportion of GERD-related NCCP diagnosed from EGD and/or $24 \mathrm{hr}$ esophageal $\mathrm{pH}$ monitoring was $30 \%$. Recently, using this same design in the same geography and ethnicities, we reported that the proportion of GERD-related NCCP diagnosed from EGD and/or 24 hr esophageal pH monitoring in the average aged subjects was $41 \%{ }^{7}$ Comparing with this previous data, our present ratio, interestingly, tends to be low. This suggests that there may be more possibilities of other pathophysiological mechanisms except GERD in the young aged NCCP, for example, visceral hyperalgesia, psychiatric disturbances, and so on. Recent studies of the biomechanical properties of the esophagus and esophageal sensation demonstrated that aging was associated with a larger lumen and a stiffer but less sensitive esophageal wall, and a lower threshold for pain and discomfort was noticed in young patients. ${ }^{16}$

The relationship to esophageal symptoms for motility abnormalities may be even more problematic. These are reflected by the American Gastroenterological Association guidelines on esophageal manometry, which recommended that it should not be used as the initial test for the evaluation of patients with $\mathrm{NCCP}^{25}$ In our study, non GERD-associated esophageal motility disorders were found in only $10 \%$.

Also, we examined the effect of PPI testing, in patients with weekly NCCP or more than weekly. Several observations have confirmed the usefulness of PPI therapy for the diagnosis of NCCP. ${ }^{11,12,14}$ The PPI test is a simple, non-invasive diagnostic tool for GERD. It is readily available at the disposal of primary care physicians and increases their role in evaluating and treating patients with the spectrum of GERD. It offers significant cost savings when compared to other diagnostic tests. In our previous data, that is $81 \%$ of positive PPI test for the GERD in NCCP patients, it has been demonstrated that 2 weeks of high-dose PPI is a simple and clinically practical method for diagnosing GERD in Korea, as well. ${ }^{14}$ While, in the present trial, the lansoprazole trial resulted in symptom improvement in $89 \%$ of GERD-related NCCP patients and this result was statistically significant compared with non GERD-related NCCP patients. Comparing with our previous data, this ratio tends to be similar or rather high. This result suggests that an empirical trial with a high-dose PPI is a simple and accurate method for diagnosing GERD-related NCCP in young patients with NCCP, as well.

Our present study has several important limitations. This study design did not include the average aged patients with NCCP and a placebo control for PPI test. However, there are 
some important and consistent findings, comparing with our previous average aged subject studies for NCCP with the same geography and ethnicities and the similar sample size and frame. In young aged NCCP patients, the proportion of GERD-related NCCP tended to be less frequent, in spite of no comparative analysis of the average aged NCCP patients. An empirical trial with a high-dose PPI during 2 weeks was highly diagnostic for patients with GERD-related NCCP in young aged NCCP patients, therefore, PPI test may assist either directly or supplementary to the physician's clinical judgment of young aged NCCP, preferably.

To accurately comprehend the differences of the prevalence of GERD according to age in patients with NCCP, their systemized symptomatic characteristics, and the clinical usefulness of the empirical trial with PPI, a large-scale investigation with case-control comparative design for young aged and average aged NCCP patients should be considered in the near future.

\section{References}

1. Faybush EM, Fass R. Gastroesophageal reflux disease in noncardiac chest pain. Gastroenterol Clin North Am 2004;33:41-54.

2. Eslick GD, Fass R. Noncardiac chest pain: evaluation and treatment. Gastroenterol Clin North Am 2003;32:531-552.

3. Eslick GD, Jones MP, Talley NJ. Non-cardiac chest pain: prevalence, risk factors, impact and consulting-a population-based study. Aliment Pharmacol Ther 2003;17:1115-1124.

4. Locke GR 3rd, Talley NJ, Fett SL, Zinsmeister AR, Melton LJ 3rd. Prevalence and clinical spectrum of gastroesophageal reflux: a population-based study in Olmsted County, Minnesota. Gastroenterology 1997;112:1448-1456.

5. Chambers J, Bass C. Chest pain with normal coronary anatomy: a review of natural history and possible etiologic factors. Prog Cardiovasc Dis 1990;33:161-184.

6. Eslick GD, Talley NJ. Non-cardiac chest pain: predictors of health care seeking, the types of health care professional consulted, work absenteeism and interruption of daily activities. Aliment Pharmacol Ther 2004;20:909-915.

7. Kim JH, Rhee PL, Park EH, Son HJ, Kim JJ, Rhee JC. Clinical usefulness of subgrouping of patients with non-cardiac chest pain according to characteristic symptoms in Korea. J Gastroenterol Hepatol 2007;22:320-325.

8. Pandak WM, Arezo S, Everett S, et al. Short course of omeprazole: a better first diagnostic approach to noncardiac chest pain than endoscopy, manometry, or 24-hour esophageal $\mathrm{pH}$ monitoring. J Clin
Gastroenterol 2002;35:307-314

9. Wong WM, Lai KC, Lau CP, et al. Upper gastrointestinal evaluation of Chinese patients with non-cardiac chest pain. Aliment Pharmacol Ther 2002;16:465-471.

10. Richter JE. Chest pain and gastroesophageal reflux disease. J Clin Gastroenterol 2000;30(suppl 3):S39-S41.

11. Fass R, Fennerty MB, Ofman JJ, et al. The clinical and economic value of a short course of omeprazole in patients with noncardiac chest pain. Gastroenterology 1998;115:42-49.

12. Cremonini F, Wise J, Moayyedi P, Talley NJ. Diagnostic and therapeutic use of proton pump inhibitors in non-cardiac chest pain: a metaanalysis. Am J Gastroenterol 2005;100:1226-1232.

13. Wang WH, Huang JQ, Zheng GF, et al. Is proton pump inhibitor testing an effective approach to diagnose gastroesophageal reflux disease in patients with noncardiac chest pain?: a meta-analysis. Arch Intern Med 2005;165:1222-1228.

14. Kim JH, Sinn DH, Son HJ, Kim JJ, Rhee JC, Rhee PL. Comparison of one-week and two-week empirical trial with a high-dose rabeprazole in non-cardiac chest pain patients. J Gastroenterol Hepatol 2009;24:1504-1509.

15. Eslick GD. Noncardiac chest pain: epidemiology, natural history, health care seeking, and quality of life. Gastroenterol Clin North Am 2004;33:1-23.

16. Rao SS, Mudipalli RS, Mujica VR, Patel RS, Zimmerman B. Effects of gender and age on esophageal biomechanical properties and sensation. Am J Gastroenterol 2003;98:1688-1695.

17. Lundell LR, Dent J, Bennett JR, et al. Endoscopic assessment of oesophagitis: clinical and functional correlates and further validation of the Los Angeles classification. Gut 1999;45:172-180.

18. Spechler SJ, Castell DO. Classification of oesophageal motility abnormalities. Gut 2001;49:145-151.

19. Jamieson JR, Stein HJ, DeMeester TR, et al. Ambulatory 24-h esophageal $\mathrm{pH}$ monitoring: normal values, optimal thresholds, specificity, sensitivity, and reproducibility. Am J Gastroenterol 1992;87:1102-1111.

20. Achem SR. New frontiers for the treatment of noncardiac chest pain the adenosine receptors. Am J Gastroenterol 2007;102:939-941.

21. Fass R, Dickman R. Non-cardiac chest pain: an update. Neurogastroenterol Motil 2006;18:408-417.

22. Achem SR, DeVault KR. Recent developments in chest pain of undetermined origin. Curr Gastroenterol Rep 2000;2:201-209.

23. Van Handel D, Fass R. The pathophysiology of non-cardiac chest pain. J Gastroenterol Hepatol 2005;20(suppl):S6-S13.

24. Nevens F, Janssens J, Piessens J, Ghillebert G, De Geest H, Vantrappen G. Prospective study on prevalence of esophageal chest pain in patients referred on an elective basis to a cardiac unit for suspected myocardial ischemia. Dig Dis Sci 1991;36:229-235.

25. Kahrilas PJ, Clouse RE, Hogan WJ. American Gastroenterological Association technical review on the clinical use of esophageal manometry. Gastroenterology 1994;107:1865-1884. 